\title{
Antiferromagnetic spin-1 Ising model. II. Interface structure and kinetic phase transition
}

\author{
Y. Saito and H. Müller-Krumbhaara) \\ Institut für Festkörperforschung der Kernforschungsanlage Jülich, D-5170 Jülich, Federal Republic \\ of Germany \\ (Received 5 May 1980; accepted 8 September 1980)
}

\begin{abstract}
The interface structure and the kinetics of a spin-1 antiferromagnetic Ising model are investigated and applied to crystal growth. For a certain range of model parameters the system undergoes two phase transitions: crystallization (nonmagnetic to magnetic) and an order-disorder (antiferromagnetic) transition of the sublattice ordering in the solid phase. Thus there are two interfaces associated with the three different phases. The structure of each interface is described by a tanh profile in a time-dependent Ginzburg-Landau approach. Various properties of the interfaces which depend on the driving force are studied. A remarkable change in structure is observed for large interface velocities: If the interface between an ordered-solid phase and a gas phase exceeds a critical velocity, a disordered-solid phase forms in between, with a width which increases proportional to time (kinetic phase transition).
\end{abstract}

\section{INTRODUCTION}

In a previous paper ${ }^{1}$ (hereafter referred to as I) one of the authors (YS) discussed the phase diagram and other bulk properties of a binary alloy in terms of an antifer romagnetic spin-1 Ising model. The model allows one to describe crystallization as well as an order-disorder transition in the solid phase. (We refer to phase with density close to one as a solid phase, and a phase with density close to zero as a gas phase.)

When the solid alloy has a surface or when it coexists with the gas, its surface or interfacial structure is not described by a bulk phase diagram, and new phenomena such as surface reconstruction and surface segregation or enrichment ${ }^{2-4}$ are expected to occur.

Another group of phenomena is associated with kinetics. When the crystal is steadily growing with the system kept in a nonequilibrium state, the composition and structure of the bulk crystal and of the interfaces may also deviate significantly from the equilibrium composition and structure.

Several investigations have been recently carried out in which the growth of a binary alloy is described phenomenologically, ${ }^{5,6}$ with computer simulations ${ }^{7-11}$ or approximate microscopic calculations. ${ }^{7,8,12-15}$ Most of those studies were concerned with an alloy without a phase transition or with phase separation in a solid phase. For alloys with an order-disorder transition in the solid phase a phenomena referred to as the "kinetic phase transition" may occur. ${ }^{7,8}$ The concept of a kinetic phase transition or kinetic disordering was originally introduced by Chernov and Lewis ${ }^{7}$ in connection with a simple model for one-dimensional growth via surface steps on a crystal. Recently, a computer simulation was performed for a binary solid-on-solid (SOS) model, but the equilibrium phase diagram for the bulk system was not discussed. ${ }^{9}$ This information is important in understanding the mechanism of disordering as induced by kinetics. In the previous paper I the spin-1 antiferromagnetic

\footnotetext{
a) Theoretische Physik der Universität Hannover, D-3000 Hannover, Fed. Rep. Germany.
}

Ising (AFI) model was used to describe a system with crystallization as well as an order-disorder transition, and its bulk phase diagram was obtained. In this second paper II we extend our investigation to the interface structure and the steady-state kinetics, and discuss the kinetic phase transition in detail.

In the next section the kinetics of the spin-1 AFI model is described in the layered mean-field approximation (LMFA). In Sec. III the interface structure and the crystal growth are described phenomenologically with a time-dependent Ginzburg-Landau approach in some simple cases. In Sec. IV some more general cases are calculated numerically, and the kinetic phase transition is discussed. The results are summarized in the last section.

\section{LAYERED MEAN-FIELD APPROXIMATION AND KINETICS}

A lattice model for a binary alloy system with crystallization and order-disorder transitions was described in $I$ in terms of a spin-1 Ising system. If the $i$ th site is occupied by an A (B) atom, the spin $S_{i}$ takes the value $1(-1)$, and if it is empty, $S_{i}$ takes the value zero. Assuming interaction energies $-J_{\mathbf{A} A},-J_{\mathbf{A B}}$, and $-J_{\mathbf{B B}}$ for the corresponding nearest-neighbor atomic pairs $\mathrm{A}-\mathrm{A}, \mathrm{A}-\mathrm{B}$, and $\mathrm{B}-\mathrm{B}$, respectively, the Hamiltonian may be written as

$$
\begin{aligned}
\mathcal{H}= & -\sum_{\langle i j\rangle}\left[\tilde{K} Q_{i} Q_{j}+\tilde{J} S_{i} S_{j}+\tilde{L}\left(S_{i} Q_{j}+Q_{i} S_{j}\right)\right] \\
& -H \sum_{i} S_{i}+\Delta \sum_{i} Q_{i},
\end{aligned}
$$

where $Q_{i}=S_{i}^{2}$. The coupling constants $\bar{K}, \tilde{J}$, and $\tilde{L}$ are related to the interaction energies $J_{\mathrm{AA}}, J_{\mathrm{AB}}$, and $J_{\mathrm{BB}}$ by

$$
\begin{aligned}
& \tilde{K}=\left(J_{\mathrm{AA}}+J_{\mathrm{BB}}+2 J_{\mathrm{AB}}\right) / 4, \\
& \tilde{J}=\left(J_{\mathrm{AA}}+J_{\mathrm{BB}}-2 J_{\mathrm{AB}}\right) / 4, \\
& \tilde{L}=\left(J_{\mathrm{AA}}-J_{\mathrm{BB}}\right) / 2,
\end{aligned}
$$

and the field variables $H$ and $\Delta$ are related to the chemi cal potential $\mu_{\mathbf{A}}$ and $\mu_{\mathrm{B}}$ of the $\mathrm{A}$ and $\mathrm{B}$ atoms, respectively, by 


$$
\begin{aligned}
& H=\left(\mu_{\mathrm{A}}-\mu_{\mathrm{B}}\right) / 2, \\
& \Delta=-\left(\mu_{\mathrm{A}}+\mu_{\mathrm{B}}\right) / 2 .
\end{aligned}
$$

Various phase diagrams for the cases of a system which undergoes an order-disorder transition $(\tilde{J}<0)$ were calculated in the mean-field approximation in $I$. For certain values of the parameters $\tilde{K}, \tilde{J}$, and $\tilde{L}$, the gas (low density phase) coexists with the disordered solid (high density phase). Depending on the values of the parameters, the order-disorder transition in the solid is either first or second order. The most in teresting case occurs where the crystallization and the order-disorder transition occur simultaneously, i. e., the ordered solid coexists with the gas. If the chemical potential $\Delta$ does not have its equilibrium value, the ordered crystal grows from the gas phase, and the relation between the growth rate of the crystal and the or dering of the alloy can be investigated. Since the chemical potential is independent of the sublattice structure, crystalline atoms attach to both sublattices $a$ and $b$ equivalently. Thus, a rapid growth rate of the crystal will lead to disordering of the alloy structure.
In order to describe the interface structure formed between two coexisting phases, we use the layered mean-field approximation (LMFA), in which one considers layers parallel to the interface. In the LMFA the state of the $n$th layer is characterized by the number of sites $N_{\mathrm{X}}^{x}(n)$ of the $x$ sublattice occupied by $\mathrm{X}$ atoms. Hereafter $\mathrm{X}$ represents the atomic species $\mathrm{A}$ or $\mathrm{B}$, and $x$ represents the sublattice species $a$ or $b$. The quantity

$$
N_{V}^{x}(n)=A / 2-N_{\mathrm{A}}^{x}(n)-N_{\mathrm{B}}^{x}(n)
$$

is the number of empty sites in the $n$th layer. Here $A$ represents the total number of sites per layer. The total number of crystal atoms (irrespective of the species A or B) on the $x$ sublattice of the $n$th layer is given by

$$
Q^{x}(n) \equiv N_{\mathrm{A}}^{x}(n)+N_{\mathrm{B}}^{x}(n) \equiv \frac{1}{2} A q^{x}(n)
$$

and the composition is defined by

$$
M^{x}(n) \equiv N_{A}^{x}(n)-N_{\mathrm{B}}^{x}(n) \equiv \frac{1}{2} A m^{x}(n) .
$$

In the LMFA the Hamiltonian (1) is written as

$$
\begin{aligned}
\mathcal{C}= & -\frac{1}{2} A \sum_{n=1}^{N}\left[\tilde{K}\left(z_{\|} q^{a}(n) q^{b}(n)+q^{a}(n) q^{b}(n+1)+q^{a}(n+1) q^{b}(n)\right)+\tilde{J}\left(z_{\sharp} m^{a}(n) m^{b}(n)+m^{a}(n) m^{b}(n+1)+m^{a}(n+1) m^{b}(n)\right)\right. \\
& +\bar{L}\left(z_{\|} m^{a}(n) q^{b}(n)+m^{a}(n) q^{b}(n+1)+m^{a}(n+1) q^{b}(n)+z_{\sharp 1} q^{a}(n) m^{b}(n)+q^{a}(n) m^{b}(n+1)+q^{a}(n+1) m^{b}(n)\right) \\
& \left.+2 H\left(m^{a}(n)+m^{b}(n)\right)-2 \Delta\left(q^{a}(n)+q^{b}(n)\right)\right] .
\end{aligned}
$$

Here $z_{\|}$is the number of nearest neighbors in the layer, e.g., four for the simple cubic lattice. $N$ is the total number of layers.

We now describe the kinetics using a stochastic master equation formulation. In the gas phase the mobility of the atoms is so high that the adsorption rate of impinging atoms from the particle bath is homogeneous and independent of the lattice site. This is not, of course, a sufficient description of the dynamics in the bulk solid. However, since the time scale of the processes in the bulk solid is long in comparison with that of interface advancement, we expect that the description is basically correct around the interface. We intend to introduce a more refined kinetic model in the future, which includes nearest-neighbor exchange processes, but for simplicity we restrict ourselves to single-site processes here.

The crystallization (or adsorption) rate is assumed to be homogeneous and only depend on the chemical potential. The evaporation rate, on the other hand, is assumed to depend on the local environment. (A kinetic process of this type is realized in the case of an adsorbed monolayer system in contact with the vapor, which works as a particle bath.) The rate of adsorption of an $\mathrm{X}$ atom $(\mathrm{X}=\mathrm{A}$ or $\mathrm{B})$ on a $x$ sublattice site $(x=a$ or $b$ ) in the $n$th layer at a temperature $T$ is given by

$$
W\left(N_{\mathrm{X}}^{x}(n)-N_{\mathrm{X}}^{x}(n)+1\right)=\frac{1}{\tau} N_{V}^{x}(n) e^{\mu} \mathrm{x}^{\prime T} .
$$

The pre-exponential factor follows from the fact that an atom can only attach to a vacant site. The evaporation process depends on the energy required to break the atomic bonds. In order to assure thermal equilibrium the rate of evaporation and adsorption should satisfy the detailed balance condition

$$
\begin{aligned}
& \frac{W\left(N_{\mathbf{X}}^{x}(n)+1-N_{\mathbf{X}}^{x}(n)\right)}{W\left(N_{\mathbf{X}}^{x}(n)-N_{\mathbf{X}}^{x}(n)+1\right)} \\
& \quad=\frac{N_{\mathbf{X}}^{x}(n)+1}{N_{V}^{x}(n)} \exp \left\{-\frac{1}{T}\left[\mathfrak{H}\left(N_{\mathbf{X}}^{x}(n)\right)-\mathcal{H}\left(N_{\mathbf{X}}^{\mathbf{X}}(n)+1\right)\right]\right\} .
\end{aligned}
$$

The pre-exponential factor takes the entropy contribution into account in the LMFA. From Eqs. (7) and (8), it follows that the transition rate $W$ from configuration $\left\{Q^{a}(n), Q^{b}(n), M^{a}(n), M^{b}(n)\right\}$ to $\left\{Q^{a}(n)+r^{a}(n), Q^{b}(n)+r^{b}(n)\right.$, $\left.M^{a}(n)+s^{a}(n), M^{b}(n)+s^{b}(n)\right\}$ with jumps $r^{a}(n), r^{b}(n), s^{a}(n)$, $s^{b}(n)=0$ or \pm 1 is an extensive quantity proportional to the size $A$ of the layer:

$$
\begin{aligned}
& W\left(Q^{a}(n), Q^{b}(n), M^{a}(n), M^{b}(n)-Q^{a}(n)+r^{a}(n), Q^{b}(n)+r^{b}(n), M^{a}(n)+s^{a}(n), M^{b}(n)+s^{b}(n)\right) \\
& \quad \equiv \frac{A}{2} w\left(q^{a}(n), q^{b}(n), m^{a}(n), m^{b}(n) ; r^{a}(n), r^{b}(n), s^{a}(n), s^{b}(n)\right) .
\end{aligned}
$$


Due to the extensive nature (9) of the transition rate, the evolution of the most probable values of the densities $q^{a}(n)$, $q^{b}(n), m^{a}(n)$, and $m^{b}(n)$, defined in Eq. (5), is described by ${ }^{16,17}$

$$
\begin{aligned}
& \frac{d}{d t} q^{x}(n)=\sum_{\tau^{a}} \sum_{r^{b}} \sum_{s^{a}} \sum_{s^{b}} r^{x}(n) w\left(q^{a}(n), q^{b}(n), m^{a}(n), m^{b}(n) ; r^{a}(n), r^{b}(n), s^{a}(n), s^{b}(n)\right), \\
& \frac{d}{d t} m^{x}(n)=\sum_{r^{a}} \sum_{r^{b}} \sum_{s^{a}} \sum_{s^{b}} s^{x}(n) w\left(q^{a}(n), q^{b}(n), m^{a}(n), m^{b}(n) ; r^{a}(n), r^{b}(n), s^{a}(n), s^{b}(n)\right) .
\end{aligned}
$$

With the transition probabilities (7) and (8) and the Hamiltonian (6) the evolution equations (10) become

$$
\begin{aligned}
\tau \frac{d}{d t} q^{a}(n)= & {\left[1-q^{a}(n)\right] e^{(-\Delta+H) / T}-\frac{1}{2}\left[q^{a}(n)+m^{a}(n)\right] \exp \left\{-\left[(K+L) \tilde{q}^{b}(n)+(J+L) \tilde{m}^{b}(n)\right] / T\right\} } \\
+ & {\left[1-q^{a}(n)\right] e^{(-\Delta-H) / T}-\frac{1}{2}\left[q^{a}(n)-m^{a}(n)\right] \exp \left\{-\left[(K-L) \tilde{q}^{b}(n)+(-J+L) \tilde{m}^{b}(n)\right] / T\right\}, } \\
\tau \frac{d}{d t} q^{b}(n)= & {\left[1-q^{b}(n)\right] e^{(-\Delta+H) / T}-\frac{1}{2}\left[q^{b}(n)+m^{b}(n)\right] \exp \left\{-\left[(K+L) \tilde{q}^{a}(n)+(J+L) \tilde{m}^{a}(n)\right] / T\right\} } \\
+ & {\left[1-q^{b}(n)\right] e^{(-\Delta-H) / T}-\frac{1}{2}\left[q^{b}(n)-m^{b}(n)\right] \exp \left\{-\left[(K-L) \tilde{q}^{a}(n)+(-J+L) \tilde{m}^{a}(n)\right] / T\right\}, } \\
\tau \frac{d}{d t} m^{a}(n)= & {\left[1-q^{a}(n)\right] e^{(-\Delta+H) / T}-\frac{1}{2}\left[q^{a}(n)+m^{a}(n)\right] \exp \left\{-\left[(K+L) \tilde{q}^{b}(n)+(J+L) \tilde{m}^{b}(n)\right] / T\right\} } \\
& +\left[1-q^{a}(n)\right] e^{(-\Delta-H) / T}-\frac{1}{2}\left[q^{a}(n)-m^{a}(n)\right] \exp \left\{-\left[(K-L) \tilde{q}^{b}(n)+(-J+L) \tilde{m}^{b}(n)\right] / T\right\}, \\
\tau \frac{d}{d t} m^{b}(n)= & {\left[1-q^{b}(n)\right] e^{(-\Delta+H) / T}-\frac{1}{2}\left[q^{b}(n)+m^{b}(n)\right] \exp \left\{-\left[(K+L) \tilde{q}^{a}(n)+(J+L) \tilde{m}^{a}(n)\right] / T\right\} } \\
& +\left[1-q^{b}(n)\right] e^{(-\Delta-H) / T}-\frac{1}{2}\left[q^{b}(n)-m^{b}(n)\right] \exp \left\{-\left[(K-L) \tilde{q}^{a}(n)+(-J+L) \tilde{m}^{a}(n)\right] / T\right\},
\end{aligned}
$$

where $K=\left(z_{11}+2\right) \tilde{K}, J=\left(z_{11}+2\right) \tilde{J}$, and $L=\left(z_{11}+2\right) \tilde{L}$. The variables $\tilde{q}^{x}(n)$ and $\tilde{m}^{x}(n)$, where $x=a$ or $b$, contain spatial variations in the form

$$
\begin{aligned}
& \tilde{q}^{x}(n)=\left[z_{\|} q^{x}(n)+q^{x}(n+1)+q^{x}(n-1)\right] /\left(z_{\|}+2\right), \\
& \tilde{m}^{x}(n)=\left[z_{\|} m^{x}(n)+m^{x}(n+1)+m^{x}(n-1)\right] /\left(z_{\|}+2\right) .
\end{aligned}
$$

The stationary and homogeneous (equilibrium) solutions of Eqs. (11) agree with the solution obtained by the pressure maximization discussed in I. Regarding the low - and high-density phases as gas and solid, respectively, one finds three phases, namely, gas, disordered solid, and ordered solid. Phase diagrams describing the bulk phase of the alloy are obtained for various cases in $I$.

If two phases coexist, an interface is formed in between. The interfacial structure is characterized by space dependent variables, such as the crystal density $q(n)$, the sublattice difference of crystallization $q_{s}(n)$, the composition $m(n)$, and the sublattice ordering $m_{s}(n)$, which are defined by

$$
\begin{aligned}
& q(n)=\left[q^{a}(n)+q^{b}(n)\right] / 2, \\
& q_{s}(n)=\left[q^{a}(n)-q^{b}(n)\right] / 2, \\
& m(n)=\left[m^{a}(n)+m^{b}(n)\right] / 2, \\
& m_{s}(n)=\left[m^{a}(n)-m^{b}(n)\right] / 2,
\end{aligned}
$$

respectively. The equilibrium interface profile can be obtained from the stationary but space dependent solution of Eqs. (11). Under nonequilibrium conditions, when the crystal is growing, the interface moves with a finite velocity to crystallize the system. Since Eqs. (11) is a coupled, nonlinear, differential-difference equa- tion, the full solution can only be obtained numerically. In the next section we first consider some qualitative features of the interface structure and of the crystal growth in a simple approximate treatment.

\section{TIME-DEPENDENT GINZBURG-LANDAU MODEL}

By solving Eqs. (11) for the stationary, homogeneous case, various phase diagrams were obtained for the case $L=0$ in paper $I$. In this section the space and time dependence of the interface structure is considered in a time-dependent Ginzburg-Landau (TDGL) approach in two simple cases with a single order parameter.

The first example is the interface between the gas and the disordered solid. For simplicity the chemical potentials $\mu_{\mathbf{A}}$ and $\mu_{\mathbf{B}}$ are assumed to be equal, so that $H=0$. In this case the system is described by a single order parameter $q$ and is equivalent to a ferromagnetic spin $-\frac{1}{2}$ Ising model. The variable $\sigma$ defined by

$$
\sigma=q-\frac{1}{2}
$$

is assumed to evolve according to the TDGL equation

$$
\frac{\partial}{\partial t} \sigma(z, t)=-\frac{1}{\tau_{1}} \frac{\delta G[\sigma]}{\delta \sigma(z, t)},
$$

with the free energy functional $G[\sigma]$ given by

$G[\sigma]=\int d z\left[\frac{1}{2}\left(\frac{\partial \sigma}{\partial z}\right)^{2}-\frac{1}{2} r \sigma^{2}+\frac{1}{4} u \sigma^{4}-c\left(\Delta_{D B-G}-\Delta\right) \sigma\right]$.

Here $\tau_{1}$ defines the time scale and the coefficients $r, u$, and $c$ are positive constants. The deviation of the chemical potential $\Delta$ from the coexistence value $\Delta_{D s-G}$ 
of the gas and the disordered solid drives the crystal growth. We assume $\Delta_{\mathrm{DS}-\mathrm{G}}-\Delta$ to be small and perform calculations to first order in $\Delta_{\mathrm{DS}-\mathrm{G}}-\Delta$. The following stationary homogeneous solutions of $\mathrm{Eq}$. (15) describe the bulk-solid and gas phases:

$$
\sigma_{\mathrm{DS}, \mathrm{G}}= \pm \sqrt{r / u}+c\left(\Delta_{\mathrm{DS}-\mathrm{G}}-\Delta\right) / 2 r \text {. }
$$

From the free energy (16), one sees that the solid phase is more stable than the gas for $\Delta<\Delta_{\mathrm{DS}-\mathrm{G}}$, and the gas phase is more stable for $\Delta>\Delta_{D S-G}$. With the boundary condition

$$
\sigma(z=-\infty, t)=\sigma_{\mathrm{DS}}, \quad \sigma(z=+\infty, t)=\sigma_{\mathrm{G}},
$$

the steady -state solution of Eq. (15) is obtained as $\sigma(z, t)=\frac{1}{2}\left(\sigma_{\mathrm{DS}}+\sigma_{\mathrm{G}}\right)-\frac{1}{2}\left(\sigma_{\mathrm{DS}}-\sigma_{\mathrm{G}}\right) \tanh \left\{\left[z-z(0)-V_{q} t\right] / \xi_{q}\right\}$.

The interface moves with a velocity

$$
V_{q}=\sqrt{9 u / 8} c\left(\Delta_{\mathrm{DS}-\mathrm{G}}-\Delta\right) / \tau_{1}
$$

from the initial position $z(0)$. The interface width is $\xi_{q}$ $=\sqrt{r / 2}$. In equilibrium $\left(\Delta=\Delta_{\mathrm{DS}-\mathrm{G}}\right)$ the tanh profile (19) of the interface agrees with that obtained by Fisk and Widom. ${ }^{18,19}$ Away from equilibrium, e.g., for $\Delta<\Delta_{D S-G}$, where the gas phase is metastable, the interface propagates with velocity $V_{a}$, preserving the tanh profile, and the system crystallizes into a solid.

Another simple case is realized when the ordered solid coexists with the disordered solid. Since the total density of the crystal atoms is almost homogeneous, the system is described by a sublattice ordering $m_{s}(z, t)$. In the TDGL approximation the evolution of $m_{s}$ is given by

$$
\frac{\partial m_{s}(z, t)}{\partial t}=-\frac{1}{\tau_{2}} \frac{\delta G\left[m_{s}\right]}{\delta m_{s}(z, t)} \text {. }
$$

Here $G\left[m_{s}\right]$ is the generalization of the free energy obtained in the Appendix of I taking the spatial variation into account:

$G\left[m_{s}\right]=\int d z\left[\frac{1}{2}\left(\frac{\partial m_{s}}{\partial z}\right)^{2}+\frac{1}{2} C_{2} m_{s}^{2}+\frac{1}{4} C_{4} m_{s}^{4}+\frac{1}{6} C_{8} m_{s}^{8}\right]$.

(This expression does not allow for a crystallization transition.) The coefficients $C_{2}, C_{4}$, and $C_{6}$ are functions of the temperature $T$, and of the chemical potentials $H$ and $\Delta$. If $C_{2}=C_{2 O D}>0, C_{4}=C_{4 O D}<0$, and $C_{8}=C_{6 O D}>0$ at $\Delta=\Delta_{O S}-D s$ and satisfy the relation

$$
16 C_{2 \mathrm{OD}} C_{6 \mathrm{OD}}=3 C_{4 \mathrm{OD}}^{2},
$$

the homogeneous state without sublattice ordering $\left(m_{s}\right.$ $=0$ ) coexists with the ordered state $m_{s}=m_{s 0 \mathrm{D}}$, where

$$
m_{S O D}^{2}=3\left|C_{4 O D}\right| / 4 C_{B O D} \text {. }
$$

When the chemical potential $\Delta$ deviates from $\Delta_{O S-D S}$ the coefficients $C_{2}, C_{4}$, and $C_{6}$ also deviate from their equilibrium values

$$
\Delta_{i}=C_{i}-C_{i \circ \mathrm{D}} \quad(i=2,4,6),
$$

which lead to slight changes in the ordered state. To first order in the $\Delta_{i}$ 's, the ordered state is given by

$$
m_{s, \mathrm{st}}^{2}=m_{s O \mathrm{D}}^{2}+\left(\frac{2 \Delta_{2}}{C_{4 O \mathrm{D}}}-\frac{3 \Delta_{4}}{2 C_{6 O \mathrm{D}}}+\frac{9 C_{4 O D} \Delta_{6}}{8 C_{6 O D}^{2}}\right)
$$

and is more stable than the disordered phase $\left(m_{s}=0\right)$ for $\Delta<\Delta_{\text {Os-DS }}$. With the boundary condition

$$
m_{s}(z=-\infty, t)=m_{\mathbf{s} \mathbf{s t}}, \quad m_{s}(z=+\infty, t)=0,
$$

appropriate for the coexistence of the ordered and disordered solids, the steady-state solution of the TDGL equation (21) is

$$
m_{s}^{2}(z, t)=\frac{1}{2} m_{s, \text { st }}^{2}\left\{1-\tanh \left[z-z(0)-V_{m_{s}} t\right] / \xi_{m_{s}}\right\} .
$$

The interface between the ordered and disordered solid moves with a velocity

$V_{m_{s}}=\frac{1}{\tau_{2} \xi_{m_{s}} r_{s O D}^{2}}\left(\frac{8 \Delta_{2}}{C_{4 O D}}-\frac{3 \Delta_{4}}{C_{6 O D}}+\frac{3 C_{4 O D} \Delta_{6}}{2 C_{6 O D}^{2}}\right) \propto \Delta_{O S-D S}-\Delta$.

The interface width is given by

$$
\xi_{m_{s}}=\left(6 C_{2 O D}\right)^{-1 / 2}+O\left(\Delta_{\mathrm{OS}-\mathrm{DS}}-\Delta\right) \text {. }
$$

The change in the chemical potential $\Delta$ affects the stationary value $m_{s, s t}$, the velocity $V_{m_{s}}$, and the width $\xi_{m_{s}}$, but the tanh profile (28) of the interface is preserved.

A Ginzburg-Landau treatment of the interface between the ordered-solid and the gas phases would require a free-energy functional of both the density $q$ and the sublattice ordering $m_{s}$ and the solution of two coupled TDGL equations similar to Eqs. (15) and (21). We leave this case to a future analysis. In the following section we solve Eqs. (11) numerically for the two simple cases considered above and check the validity of the TDGL approach. Finally, we investigate the general case of the ordered-solid-gas interface where the crystallization and ordering interfere with each other.

\section{NUMERICAL SOLUTION}

In order to solve the evolution equations (11), we have to define boundary and initial conditions for a system with length $N+2$, where $N$ is a sufficiently large number. Near the boundaries the density variations are assumed to be smooth:

$$
q^{x}(0)=q^{x}(1), \quad m^{x}(0)=m^{x}(1)
$$

and

$$
q^{x}(N+1)=q^{x}(N), \quad m^{x}(N+1)=m^{x}(N),
$$

where $x$ indicates sublattices $a$ or $b$. The densities $q^{x}(n)$ and $m^{x}(n)$ for $n=1, \ldots, N$ evolve according to Eqs. (11). The initial condition for two coexisting phases 1 and 2, i.e.,

$$
\begin{array}{lll}
q^{x}(n)=q_{1}^{x}, & m^{x}(n)=m_{1}^{x}, & \text { for } 1 \leq n \leq N / 2, \\
q^{x}(n)=q_{2}^{x}, & m^{x}(n)=m_{2}^{x}, & \text { for } N / 2<n \leq N,
\end{array}
$$

is assumed, where $q_{1}^{x}, q_{2}^{x}$ and $m_{1}^{x}, m_{2}^{x}$ are the equilibrium values of the densities and compositions of phases 1 and 2 , respectively. With the steady-state profile of interfaces (19) and (28) in mind, the interface position for the crystal density $q(n)$ and the sublattice ordering $m_{s}(n)$ are defined by

$$
\bar{n}_{a}=\sum_{n=1}^{N}[q(n)-q(N)] /[q(1)-q(N)]+\frac{1}{2}
$$


and

$$
\bar{n}_{m_{s}}=\sum_{n=1}^{N}\left[m_{s}^{2}(n)-m_{s}^{2}(N)\right] /\left[m_{s}^{2}(1)-m_{s}^{2}(N)\right]+\frac{1}{2}, .
$$

respectively. A measure for the interface width is given by

$$
\begin{aligned}
& 2 \bar{\xi}_{q}=[q(1)-q(N)] /\left[q\left(\left[\bar{n}_{q}\right]\right)-q\left(\left[\bar{n}_{q}\right]+1\right)\right], \\
& 2 \bar{\xi}_{m_{s}}=\left[m_{s}^{2}(1)-m_{s}^{2}(N)\right] /\left[m_{s}^{2}\left(\left[\bar{n}_{m_{s}}\right]\right)-m_{s}^{2}\left(\left[\bar{n}_{m_{s}}\right]+1\right)\right],
\end{aligned}
$$

where the notation $[a]$ above means the integer part of the real number $a$. The system length $N$ should be large compared with the widths $2 \bar{\xi}_{q}$ and $2 \bar{\xi}_{m_{s}}$.

When the chemical potential $\Delta$ is different from the equilibrium value, the system grows, and the interfaces propagate. The propagation velocities of the interfaces $\bar{V}_{q}$ and $\bar{V}_{m_{s}}$ are defined as

$$
\bar{V}_{q}=d \bar{n}_{q} / d t=[q(1)-q(N)]^{-1} \sum_{n=1}^{N} d q(n) / d t,
$$

and

$$
\bar{V}_{m_{s}}=d \bar{n}_{m_{s}} / d t=\left[m_{s}^{2}(1)-m_{s}^{2}(N)\right]^{-1} \sum_{n=1}^{N} d m_{s}^{2}(n) / d t,
$$

respectively. In order to follow the motion of the interface of the crystal density for example, we keep $\bar{n}_{q}$ in the central region of the system $N / 2 \leq \bar{n}_{q}<N / 2+1$. When $\bar{n}_{q}$ leaves this region, the system is shifted so as to keep the interface in the center. For a sufficiently long system $N \gg 2 \bar{\xi}_{q}$, the effect of shifting, which affects the boundaries, can be neglected.

The phase diagram in paper I show various combinations of coexisting phases. In what follows three typical cases are considered: (A) gas-disordered-solid coexistence, (B) ordered-solid-disordered-solid coexistence, and (C) gas-ordered-solid coexistence. The first two cases are described by the single order parameters (A) crystal density $q$ and (B) sublattice ordering $m_{s}$, respectively, while the last case (C) requires both order parameters $q$ and $m_{s}$.

\section{A. Growth of the disordered solid from the gas}

For a system with coupling constants $K=3.8, J=-1.0$, $L=0$ and $H=0, T=0$, there is a certain region of $\Delta$ where
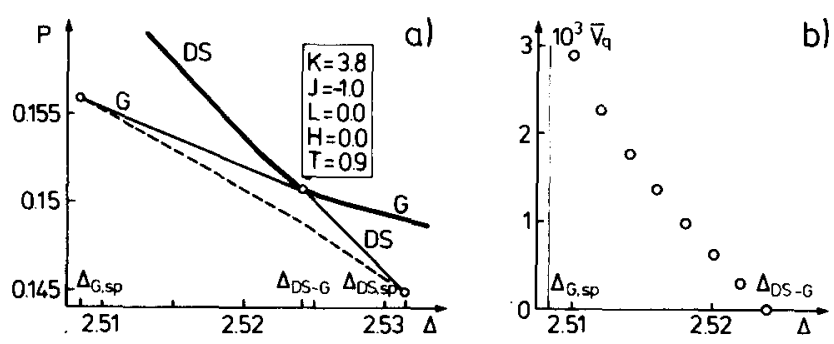

FIG. 1. (a) The pressure $P$ as a function of $\Delta$ for the case of a gas (G) coexisting with a disordered solid (DS). The thick lines represent the most stable phases, the thin lines metastable phases, and the dashed lines unstable phases. The gas-disordered solid coexistence takes place at $\Delta_{\mathrm{DS}-\mathrm{G}} \cdot \Delta_{\mathrm{G}, \text { s }}$ and $\Delta_{\mathrm{DS}, \mathrm{so}}$ represent the spinodal values of the gas and the disordered solid phases, respectively. (b) The interfacial velocity of the crystal density $\bar{V}_{q}$ as a function of $\Delta$.
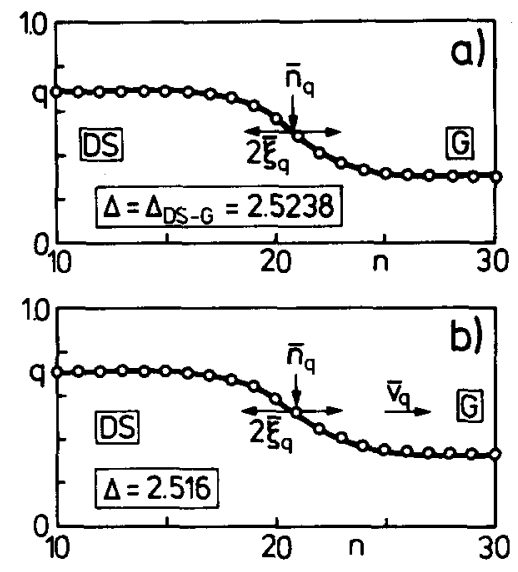

FIG. 2. The interfacial profiles of the crystal density $q(n)$ for the system described in Fig. 1.

Eqs. (11) has two stationary homogeneous solutions, namely, the gas (G) and the disordered-solid (DS) phases. On maximizing the pressure $P$, defined by Eq. (13) in I and shown in Fig. 1(a), the gas (disordered-solid) phase is seen to be more stable for $\Delta>(<) \Delta_{\mathrm{Ds}-\mathrm{G}}$, and the two

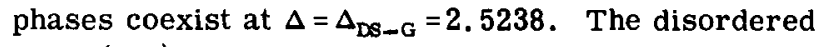
solid (gas) is metastable in the spinodal region $\Delta_{\mathrm{DE}}$, sD $>\Delta>\Delta_{\mathrm{Ds}-\mathrm{G}}\left(\Delta_{\mathrm{DS}-\mathrm{G}}>\Delta>\Delta_{\mathrm{G}, \text { sp }}\right)$. We first consider the equilibrium interface $\left(\Delta=\Delta_{D S-G}\right)$. Since there is no sublattice ordering, the system is characterized by the crystal density $q(n)$ as shown in Fig. 2(a) for a system of length $N=40$. (Only the central part is shown.) The interface position and width calculated by Eqs. (33) and (34) are indicated by the arrows. If $\Delta$ is lowered from the coexistence point $\Delta_{\mathrm{DS}-\mathrm{G}}$, the interface moves so as to crystallize the whole system in order to approach the true equilibrium state (i.e., uniform solid phase). The interface profile for $\Delta$ smaller than $\Delta_{D S-G}$ is plotted in Fig. 2(b). The interface moves steadily to the right to crystallize the system, but the profile itself remains almost unchanged relative to Fig. 2(a). The tanh profile of the interface [Eq. (19)]

$$
q(n)=\frac{1}{2}[q(1)+q(N)]-\frac{1}{2}[q(1)-q(N)] \tanh \left[n-\bar{n}_{q}(t)\right] / \bar{\xi}_{q},
$$

which is indicated by a continuous line in Figs. 2(a) and $2(\mathrm{~b})$, fits the numerically calculated profile very well. The velocity of the interface $\bar{V}_{Q}$ calculated ac cording to Eq. (35a) is plotted as a function of the chemical potential $\Delta$ in Fig. $1(\mathrm{~b})$. The velocity $\bar{V}_{q}$ is proportional to the driving force $\Delta_{\mathrm{DS}-\mathrm{G}}-\Delta$ for small values, but there is a nonlinear enhancement near the spinodal point $\Delta_{\mathrm{G}}$, sp associated with the instability of the gas phase. The gas phase does not exist for $\Delta<\Delta_{\mathrm{G}, \text { sp }}$.

\section{B. Growth of the ordered solid from the disordered solid}

The system is defined by the parameter values $K=2.88$, $J=-1.0, L=0$ and $H=0.6, T=0.65$. For chemical potential $\Delta$ around $\Delta_{O D-D S}=1.9589$, two phases, the or dered-solid (OS) and the disordered-solid (DS) phases, are possible. From the pressure comparison shown in Fig. 3(a), the ordered (disordered) solid is seen to be more stable for $\Delta<(>) \Delta_{\text {os-Ds }}$. Two phases coexist at 

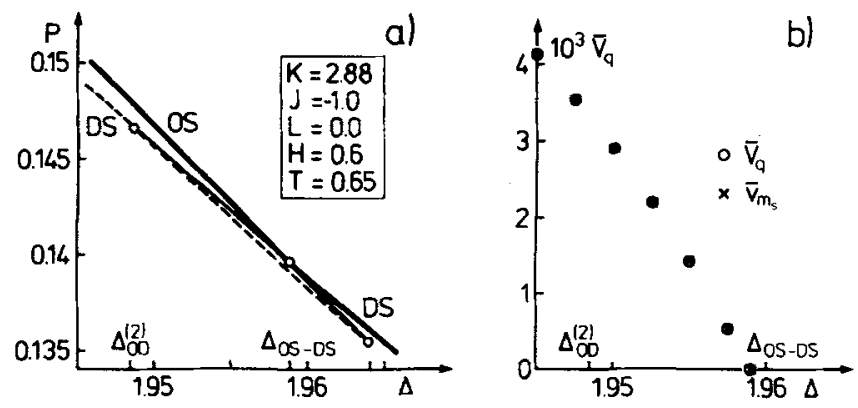

FIG. 3. (a) The pressure $P$ as a function of $\Delta$ for the case of a disordered solid (DS) coexisting with an ordered-solid (OS) phase. The first-order order-disorder transition takes place at $\Delta_{\mathrm{OS}-\mathrm{DS}}$, and $\Delta_{\mathrm{OD}}^{(2)}$ represents the bifurcation of the ordered solid from the disordered solid. (b) The interfacial velocities of the crystal density $\bar{V}_{\sigma}$ and the sublattice ordering $\bar{V}_{m_{s}}$ as a function of $\Delta$.

$\Delta=\Delta_{\text {SS-DS }}$. [The concentrations are $y_{\mathrm{OS}}=0.353$ and $y_{\text {DS }}=0.416$, and pressure $P=0.1393$. See Fig. $8(\mathrm{~d})$ of paper I.] The interface profile is calculated by integrating Eqs. (11) numerically. The equilibrium profile $\left(\Delta=\Delta_{\mathrm{Os}-\mathrm{Ds}}\right)$ is shown in Fig. $4(\mathrm{a})$. The particle density $q$ in the solid phase remains almost homogeneous, while the sublattice ordering $m_{s}$ changes rapidly across the interface. With the interface position and width calculated by Eqs. (33) and (34), the sublattice ordering $m_{s}$ fits the tanh profile

$$
m_{s}^{2}(n)=\frac{1}{2} m_{s}^{2}(1)\left\{1-\tanh \left[n-\bar{n}_{m_{s}}(t)\right] / \bar{\xi}_{m_{s}}\right\},
$$

shown by the continuous line in Fig. 4 (a) very well. On decreasing $\Delta$ the interface proceeds to the right to order the system as shown in Fig. $4(\mathrm{~b})$. The velocity of the interface of the density change $\bar{V}_{q}$ is extremely close to that of the interface between the order-disorder states $\bar{V}_{m}$ as shown in Fig. 3(b). The interfacial velocities are found to be proportional to the chemical potential deviation $\Delta_{\text {Os-Ds }}-\Delta$. At $\Delta=\Delta_{0 \mathrm{D}}^{(2)}$ the coefficient $C_{2}$ of the quadratic term of the free energy expansion $G\left[m_{3}\right]$, given by Eq. (22) becomes zero (see also the Appendix in paper, I), and for $\Delta<\Delta_{O D}^{(2)}$ the disordered-solid phase
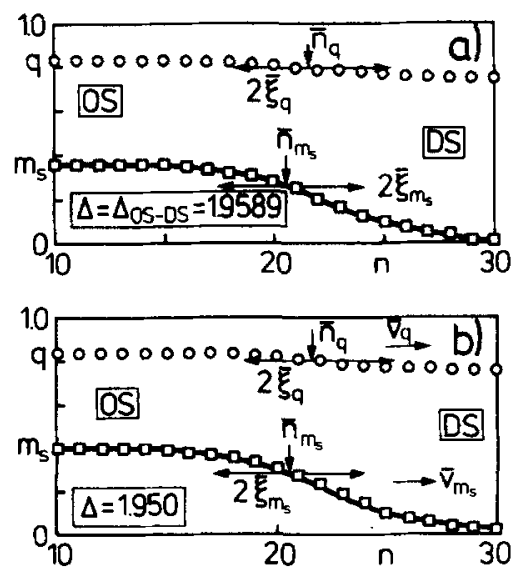

FIG. 4. The interfacial profiles of the crystal density $q(n)$ (circles) and the sublattice ordering $m_{s}(n)$ (squares) for the system described in Fig. 3.
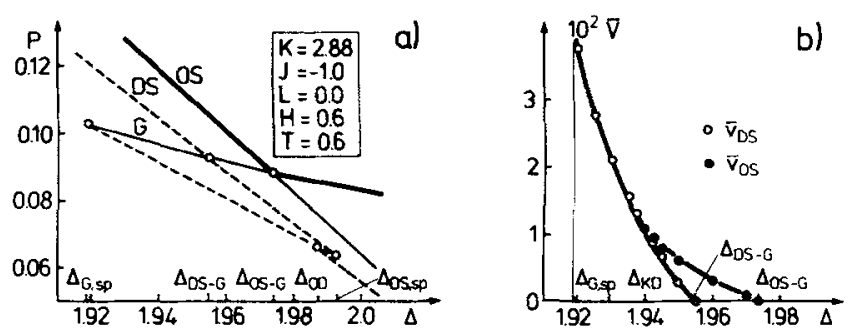

FIG. 5. (a) The pressure $P$ versus $\Delta$ for the cases of an ordered solid (OS) coexisting with a gas phase (G) in equilibrium. The disordered-solid phase (DS) is more stable than the gas for $\Delta<\Delta_{\mathrm{DS}-\mathrm{G}}$, but is always less stable than the ordered-solid phase. (b) The interface velocities of the ordered solid $\left(\bar{V}_{\text {Os }}\right)$ and of the disordered solid $\left(\bar{V}_{\mathrm{DS}}\right)$. The ordered solid growth is only possible for $\Delta$ 's larger than the value $\Delta_{\mathrm{KD}} \approx 1.94$ of the kinetic disordering.

is unstable. However, since the boundary condition favors the disordered-solid phase at the right end and since the disordered phase is one of the stationary homogeneous solutions of Eqs. (11), the interfacial velocity shows no anomalous behavior near $\Delta_{0 \mathrm{D}}^{(2)}$.

\section{Growth of the ordered solid from the gas phase}

The system is defined by the parameter values $K=2.88$, $J=-1.0, L=0$ and $H=0.6, T=0.6$ [see Fig. 5(a)]. For a certain range of $\Delta$, there are three homogeneous stationary solutions of the evolution (11), corresponding to the gas, the disordered-solid, and the ordered-solid phases. From the pressure comparison shown in Fig. 5 (a), the true equilibrium phase is seen to be either a gas for $\Delta>\Delta_{\mathrm{bs}-\mathrm{G}}$, or an ordered-solid phase for $\Delta$ $<\Delta_{\mathrm{OS}-\mathrm{G}}$. The gas phase is metastable in the region $\Delta_{\mathrm{G}, s \mathrm{~s}}<\Delta<\Delta_{\mathrm{OS}-\mathrm{G}}$, whereas the ordered solid is metastable for $\Delta_{\mathrm{os}-\mathrm{G}}<\Delta<\Delta_{\mathrm{Os}, \mathrm{sp}}$ (not shown). The disordered solid phase is always less stable than the ordered solid, but in the region $\Delta>\Delta_{\mathrm{Ds}-\mathrm{G}}$ it is more stable than the gas phase. We now consider the equilibrium interface structure between the ordered-solid and gas phases at $\Delta$ $=\Delta_{\mathrm{OS}-\mathrm{G}}=1.9735$. (The concentrations are $y_{\mathrm{OS}}=0.282$, $y_{\mathrm{G}}=0.646$.) At this point the interface has the structure shown in Fig. 6(a), and as the density $q$ decreases from the ordered solid to the gas, the sublattice ordering $m_{s}$ also decreases. The two interfaces (for $q$ and $m_{s}$ ) are tied together and behave like a single interface with a more complicated structure. This picture remains valid if $\Delta$ is decreased from the coexistence point Aos-G.

The double interface moves at a speed $\bar{V}_{a}=\bar{V}_{m_{s}}=\bar{V}_{O S}$ which is almost proportional to the deviation $\Delta_{\mathrm{os}-G}-\Delta$, as is shown in Fig. $6(\mathrm{~b})$. However, if $\Delta$ is decreased below $\Delta_{\mathrm{KD}} \simeq 1.94$, the double interface splits up into a $q$ interface, which moves with velocity $\bar{V}_{q} \equiv \overline{\boldsymbol{V}}_{\underline{\text { Ds }}}$ and an $m_{s}$ interface, which moves at a slower rate $\bar{V}_{m_{s}}$, and a disordered-solid region grows between the two interfaces. Additional information about the kinetic disordering is presented in Fig. 5(b). By choosing a boundary condition which favors the coexistence of the disordered-solid and the gas phases, we obtain the lower branch of the $\bar{V}_{\text {DS }}$ curve by starting at $\Delta=\Delta_{\text {DS-G }}[$ see 

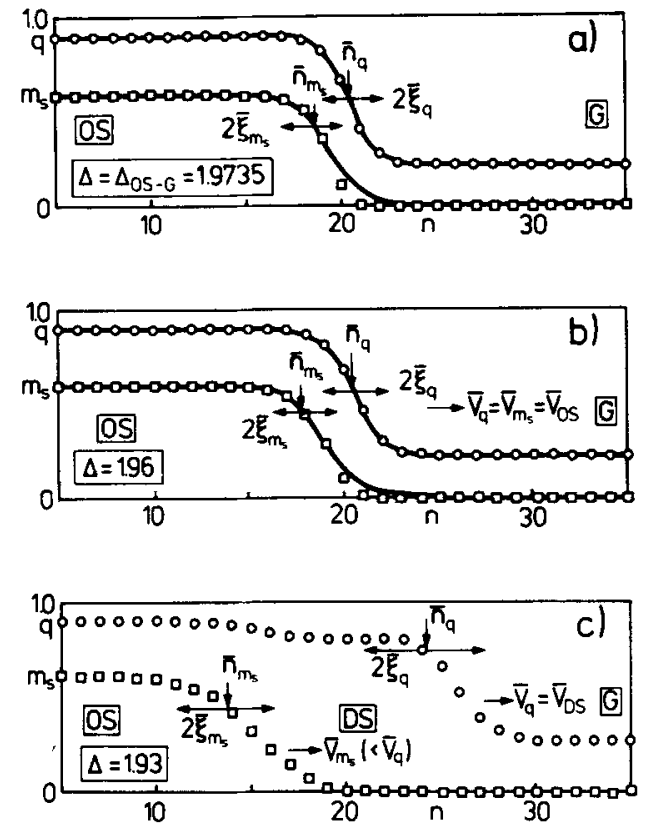

FIG. 6. The interface profile of the system described in Fig. 5. Figures $6(a)$ and $6(b)$ show the steady-state profiles, and Fig. $6(\mathrm{c})$ is a snap shot profile. In (c) the separation between the interfacial positions $\bar{n}_{q}$ and $\bar{n}_{m s}$ increases with time, and the disordered solid phase forms in between.

Fig. 5(a).] The growth rate $\bar{V}_{D S}$ is smaller than the growth rate of the ordered-solid phase $\bar{V}_{\mathrm{OS}}$ in the region $\Delta>\Delta_{K D}$. At $\Delta_{K D}$ the two rates become equal, and for $\Delta<\Delta_{K D}$ the ordered solid becomes dynamically unstable.

The above discussion gives a rather clear picture of the dynamics of kinetic disordering. However, more explicit formulation in terms of a TDGL theory would be desirable. Since the good agreement between the TDGL approach and the numerical results in the simple cases (A) and (B) considered here is very encouraging, we hope to continue our work in this direction in the future.

\section{SUMMARY}

Using a spin-1 antifer romagnetic Ising model, we have analyzed the growth of a crystal with sublattice ordering. The interface structure of the crystal density of the sublattice ordering can be well represented by the simple TDGL approximation and has a tanh profile. The interfacial velocity $\bar{V}_{q}$ is proportional to the chemi- cal potential differences $\Delta_{s-G}-\Delta$ or $\Delta_{D S-G}-\Delta$ for small deviations, but diverges at the spinodal point of the gas phase $\Delta=\Delta_{\mathrm{G}, \text { sp }}$. The sublattice ordering in the solid phase shows a kinetic anomaly. The dynamical coexistence of the gas and the ordered-solid phases during growth becomes unstable when the velocity of the interface between the gas and the disordered solid exceeds that between the gas and the ordered solid. The system crystallizes rapidly, but the sublattice ordering can not follow that speed, and the entire system is kinetically disordered. The chemical potential $\Delta_{\mathrm{KD}}$ at which kinetic disordering begins can be sharply localized as in previous models for step growth. ${ }^{7,8}$ The kinetic disordering looks similar to an equilibrium phase transition, but the transition from the ordered to the disordered solid leaves the solid in a metastable state. In our mean-field treatment and in practical applications this may not be a serious problem, but a more rigorous analysis would require the incorporation of relaxation times for the metastable states.

${ }^{1}$ Y. Saito, J. Chem. Phys. 74, 713 (1981).

${ }^{2}$ K. Binder, D. Stauffer, and V. Wildpaner, Acta Metall. 23, 1191 (1975).

${ }^{3}$ A. Theumann and K. H. Bennemann, Phys. Rev. B 17, 331 (1978).

${ }^{4}$ Y. S. Ng, T. T. Tsong, and S. B. McLane Jr., Phys. Rev. Lett. 42, 588 (1979).

${ }^{5}$ V. T. Borisov, Sov. Phys. Dokl. 7, 50 (1962).

${ }^{6}$ V. A. Petrovskii and V. T. Borisov, Sov. Phys. Dokl. 17, 606 (1972).

${ }^{7}$ A. A. Chernov, J, Lewis, J. Phys. Chem. Solids 28,2185 (1967).

${ }^{8}$ A. A. Chernov, Sov. Phys. Usp. 13, 101 (1970).

${ }^{9}$ T. A. Cherepanova, A. V. Sirin, and V. T. Borisov, Sov. Phys. Crystallogr. 22, 147 (1977).

${ }^{10} \mathrm{~T}$. A. Cherepanova and V. F. Kiselev, Sov. Phys. Crystallogr. 24, 186 (1979).

${ }^{11}$ T. A. Cherepanova, J. P. Van der Eerden, and P. Bennema, J. Cryst. Growth 44, 537 (1978).

${ }^{12} \mathrm{M}$. Takata and A. Ookawa, J. Cryst. Growth 24, 515 (1974).

${ }^{13}$ T. A. Cherepanova, Sov. Phys. Dokl. 238, 39 (1978).

${ }^{14}$ D. E. Temkin, Sov. Phys. Crystallogr. 23, 650 (1978).

${ }^{15}$ D. E. Temkin, Sov. Phys. Dokl. 23, 423 (1978).

${ }^{16}$ R. Kubo, K. Matsuo, and K. Kitahara, J. Stat. Phys. 9, 51 (1973).

${ }^{17}$ Y. Saito and H. Müller-Krumbhaar, J. Chem. Phys. 70, 1078 (1979).

${ }^{18}$ J. W. Cahn and J. E. Hilliard, J. Chem. Phys. 28, 258 (1958).

${ }^{19}$ S. Fisk and B. Widom, J. Chem. Phys. 50, 3219 (1969). 